Research Article

\title{
Creating Values out of Internet of Things: An Industrial Perspective
}

\author{
Partha Pratim Ray \\ Department of Computer Applications, Sikkim University, 6th Mile, Gangtok, Sikkim 737102, India \\ Correspondence should be addressed to Partha Pratim Ray; ppray@cus.ac.in
}

Received 14 June 2016; Revised 13 August 2016; Accepted 8 September 2016

Academic Editor: Yasuhisa Omura

Copyright (C) 2016 Partha Pratim Ray. This is an open access article distributed under the Creative Commons Attribution License, which permits unrestricted use, distribution, and reproduction in any medium, provided the original work is properly cited.

\begin{abstract}
Internet of Things based cloud is envisaged to extract values other than the specified purpose for which it is to be utilized. It is hereby apprehended that different genre of values, rather business values, could efficiently be assimilated from the Internet of Things cloud platforms. It is also investigated to identify numerous domains of applications that are currently being associated with the similar cloud platforms. A case study on various types of value generation methods has been performed. A novel Internet of Things cloud stack is proposed to disseminate and aggregate the business values. Few research challenges are observed that shall need appropriate indulgence to generate more business values out of Internet of Things based cloud. This paper also seeks few research and industry related problems that need to be resolved. It further recommends several key parameters to the enterprise and government policymakers that should immediately be dealt with for long-term success.
\end{abstract}

\section{Introduction}

Recently, a large portion of the IT based industries is getting into a new buzz word "IoT cloud," that is, Internet of Things enabled cloud. Various forms of cloud deployment models such as public, community, private, and hybrid can be seen around the day-to-day livelihood. According to a recent report, Internet of Things (IoT) is being popularized as a business concept among the leading IT market players of the world that counts the giants like Intel, Microsoft, CISCO, Google, IBM, Samsung, Apple, Oracle, ARM, Accenture, Amazon, and so forth [1]. Similarly, global consumers are eventually developing their affinity towards full-time connectivity to the internet based commodities such as smart phones, PDAs, and tablets. In this context, new businesses policies are being intrigued by the overwhelming efficiency, business process implication, and revenue generation opportunities from the available IoT cloud solutions [2]. IDC has also estimated the market growth of IoT and enabled cloud services to reach up to $\$ 7.1$ trillion by 2020 .

Before going into the details, the definition of IoT and associated terms need to be understood. IoT may be defined as "a global infrastructure for the information society enabling advanced services by interconnecting (physical and virtual) things based on, existing and evolving, interoperable information and communication technologies" [3].

The definition of "cloud" is presented as prescribed by the NIST (National Institute of Standards and Technology) in its special publication of 7 pages (800-1457) in September 2011. According to it, "cloud computing is a model for enabling ubiquitous, convenient, on-demand network access to a shared pool of configurable computing resources (e.g., networks, servers, storage, applications, and services) that can be rapidly provisioned and released with minimal management effort or service provider interaction."

Henceforth, the novel definition of IoT cloud may be framed as "a model designed to facilitate the information society, enabling advanced services by interconnecting (physical and virtual) things based on, existing and evolving, interoperable information and communication technologies through ennoblement of ubiquitous, convenient, on-demand network access to a shared pool of configurable computing resources (e.g., networks, servers, storage, applications, and services) that can be rapidly provisioned and released with minimal management effort or service provider interaction 
that leverage the need and heterogeneous connectivity issues of the user centric things in well defined fashion."

Although IoT and cloud assisted technologies and service providers are constantly emerging into the IT market, due lack of overall knowledge about mechanism of extracting special business values from the IoT cloud platforms restricts researchers, developers, and business managers to choose a set of IoT cloud services when they are in phase with development of any product or solution utilizing IoT enabled technologies.

The goal of this paper is to provide an abstract concept towards the developers and business managers to efficiently utilize IoT cloud platforms to earn values, especially business ones. Here, value creation mechanism has been repeatedly depicted for better understanding of what it is meant for. "Value" is envisaged as performance that has been assigned to particular thing/object.

Main contributions of this paper are as follows:

(i) To identify the areas where IoT cloud service providers can generate business values in global market.

(ii) To provide a case study to make reader understand what else can be achieved from a designated "thing."

(iii) To propose an IoT cloud stack to augment the needs of more value generation from the attached devices/things with the system so as to enhance the capability of the envisaged business setup.

(iv) To point out industry based challenges to motivate the researchers to continue on the paths prescribed in this article.

(v) To recommend few new approaches so that IoT based industrial perspectives may be changed.

This article is framed as follows. Section 2 presents related works. Section 3 illustrates the various IoT cloud platforms available in current market and their association with business value creation. Section 4 describes the technologies behind the IoT cloud. A similar framework has been proposed in this section. Section 5 points out Industrial Io $\mathrm{T}$ based challenges to be taken care of by the corporate sector and scientific community. Section 6 presents various recommendations to solve these challenges in industrial perspectives. It is followed by Section 7 which concludes the paper.

\section{Related Works}

This section provides various tasks related to Io $\mathrm{T}$ and enabled cloud services where business "values" [4] are created in multiforms. Further, a linkage is been opted to establish a seamless flow for readers comprehension with previous section.

The field of applications of IoT cloud is diverse. The most promising area of application includes industry 4.0 [5] or the smart industry, where the production systems and production sites are built to be intelligent, connected, and autonomous in nature. In smart home or living area, intelligent refrigerators, security cameras, intelligent lights, doors, and even kitchen utensils are earning huge attention, while smart energy meters, water meters, and gas meters are helping in optimized usage of the consumable resources. Similarly, smart city and vehicles are counting the bus timing, identifying less traffic routes, and monitoring the status of parking space, while smart health care systems are measuring vital signs of individual $24 \times 7$, helping in taking preventive measures before the actual disease may prune itself into the body, and connecting health to user, medical facilities, and caregivers [6-9].

In defence $[10,11]$, drones and high flight balloons do surveillance over the suspect region over the earth, while in smart agriculture area, dairy farming, bee keeping, cattle monitoring, and vine yard monitoring are getting popular; further information regarding resident or remote environmental parameters are no longer difficult to be accessed. Sports area is also being assimilated with IoT cloud so as to set up a connected ecosystem [12, 13]. Model driven tree reference model [14] and generalized domain model architecture [15] are proposed to cater the architectural value creation process in IoT. Strong need for integration of cloud and IoT is mentioned in [16] where an agentoriented and cloud assisted paradigm is envisaged based on a novel reference architecture. After analyzing various depicted papers, a generic architecture is presented in [17] where an IoT supported cloud based smart device is evaluated to perform data monitoring, gathering, and processing. A brief survey of the state of the art in sensing services over cloudcentric IoT and recent challenges are mentioned aiming at defining the taxonomy of the stated surveyed schemes in [18]. CloudIoT platform [19] is proposed while highlighting the complementarity and the need for the integration of cloud and IoT together. Based on the results obtained from survey of the measurement for the wine growing season during 2014, an M2M (Machine-to-Machine) remote telemetry station in cooperation with a big data processing platform and several sensors is implemented to demonstrate the use of IoT cloud systems and big data processing in order to implement disease prediction and alerting application for viticulture [20]. Wang and Ranjan [21] describe various notions (i.e., datacenter cloud computing infrastructure service stack, data management service across datacenters, Data Intensive Workflow Computing (DIWC), benchmark, application kernels, standards, recommendations, etc.) to visualize how distributed IoT data could be processed in the clouds. An IoT based Software Defined Radio (SDR) enabled cloud computing paradigm is implemented to provide a unified view on accessing, configuring, and operating of the IoT cloud systems while implying dynamic and on-demand service frameworks [22]. Reference [23] proposes the U-GovOps, a novel framework for dynamic, on-demand governance of elastic IoT cloud systems under uncertainty while introducing a declarative policy language to simplify the development of uncertainty, and elasticity-aware governance strategies. Seven different principles of engineering IoT cloud systems are prescribed so as to comprehend and provide knowledge about how IoT cloud systems could provide a coherent software layer for continuous deployment, provisioning, and 
execution of applications for various domains [24]. An IoT cloud framework is designed to harmonize cloud-scale IoT services defining intention of user or device to enable communication between connected devices in cloud-scale IoT services [25]. Reference [26] discusses the prospective evolution of IoT clouds towards federated ecosystems, where Io $\mathrm{T}$ cloud systems cooperate to offer more flexible services by proposing a 3-layered federated IoT architecture. A framework is proposed for scalable and real-time provisioning of IoT cloud based services in smart cities. These two features have been achieved by employing a novel hierarchical model and populating them in a tree structure containing references to services and their real-time data [27]. Agent based IoT cloud computing is also provisioned to support the development of decentralized, dynamic, and cooperating open IoT cloud systems incorporating multiple IoT agents [28]. While talking about "values," temperature and humidity sensors are used to measure the thermal comfort for indoor occupants using IoT cloud as its backbone [29].

\section{IoT Cloud Platforms and Business Value Creation}

This section illustrates how IoT is being utilized in different sectors of industries. Further, a hypothetical case study is performed involving electric bulb, waste bin, and tractor to show how these "things" can give more business values other than their original and ordinary functional values.

Though IoT clouds vary in many factors, such as real-time data capture, data visualization, cloud type, data analytics, device configuration, API protocols, cost, and big data, the main purpose of IoT cloud is to facilitate intelligent integration and accessibility of the things/devices and associated surrounding ecosystem thus bridging the gap in the physicaldigital divide.

The following list includes several existing IoT cloud service providers that enable end-to-end IoT based analytics, storage, and real-time processing support to the customers and business farms: Xively, ThingSpeak, Plotly, Exosite, GroveStreams, Temboo, ThingWorx, Carriots, Nimbits, KAA, IBM IoT, Oracle Open IoT, Microsoft Research Lab of Things, SensorCloud, Ayla's IoT Cloud Fabric, Arrayent Connect TM, Aer Cloud, thethings, io, SeeControl IoT, and Jasper Control Center.

The opportunities for the IoT cloud providers are of multifold. Figure 1 illustrates various areas where corporate investments are currently being sought for. It starts with leveraging a common platform for consumer-electronics and appliances design sector, while heterogeneous Machine-toMachine (M2M) communication platform is cumulating the digital telephony among the devices, ubiquitous transportation systems are being implemented at various smart cities, smart health care facilitation gives the fully connected ambience to the patients, and environment monitoring is deliberately getting huge attention. Similarly, defence, security, and military forces seem to be keen on getting full exploration with IoT cloud using NFC (Near Field Communication) and innovative nanotechnology solutions at the war site. Smart agriculture systems are being popularized among the global

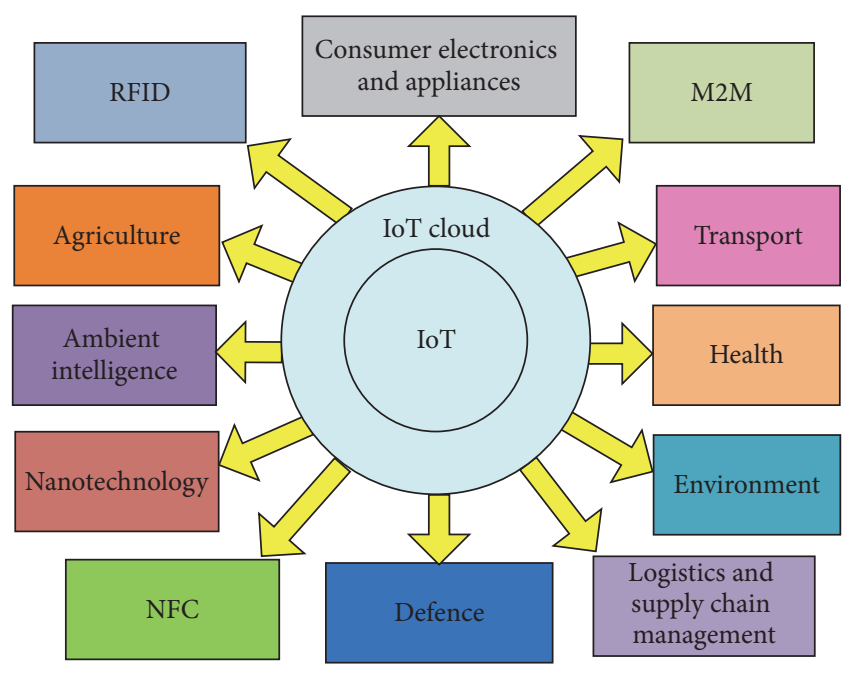

FIgURE 1: IoT cloud based investments.

farmers that use the intelligence of IoT cloud platform to result in a beginning of a new era of precision cum optimized agriculture for better yield. Ambient intelligence is another area where IoT cloud is momentarily getting itself.

The original business value of IoT cloud lies on its actual process of dissemination with things/devices to the extraction of information for some specific purposes. Value creation primarily depends on the IoT cloud stack which will be discussed in next section. Figure 2 presents the logical schema of such value creation. It illustrates that IoT cloud based solutions typically combine physical things/devices with information technology in the form of available hardware and software. IoT stack plays the crucial role in resolving these issues. IoT cloud stack is placed on top of IoT stack which results in thing-based functionalities either physical or local influence. Intelligent and assisted IoT cloud services empower user/customer/corporate farms to solicit in decision making, revenue generation, and inductive cum incumbent processes.

For the sake of understanding, let us take the activities of bulb shown in Figure 2. Normally the physical task of a bulb is to enlighten its periphery. However, when the bulb is attached to an integrated form of motion detector sensor through an IoT cloud, it shall be able to detect movement in its range. By this way, the light bulb may act as smart intrusion detection system. Further, the information of detection of any movement may be sent to the owner in form of e-mail, messages, or social status updates.

Similarly, a bin (garbage collector) is primarily used for storage of excess elements of household or office. This thingbased function may be enhanced with help of IoT cloud technology so that it can autonomously measure and monitor its current load in terms of weight, thus detecting its levels of stocks. It may further notify the municipal or community service providers to replenish its contents into the dust trays while activating GPS facilities by providing plan for automatic optimized traffic route through the busy roads of the city. Normally a tractor is meant for towing mechanical devices for 


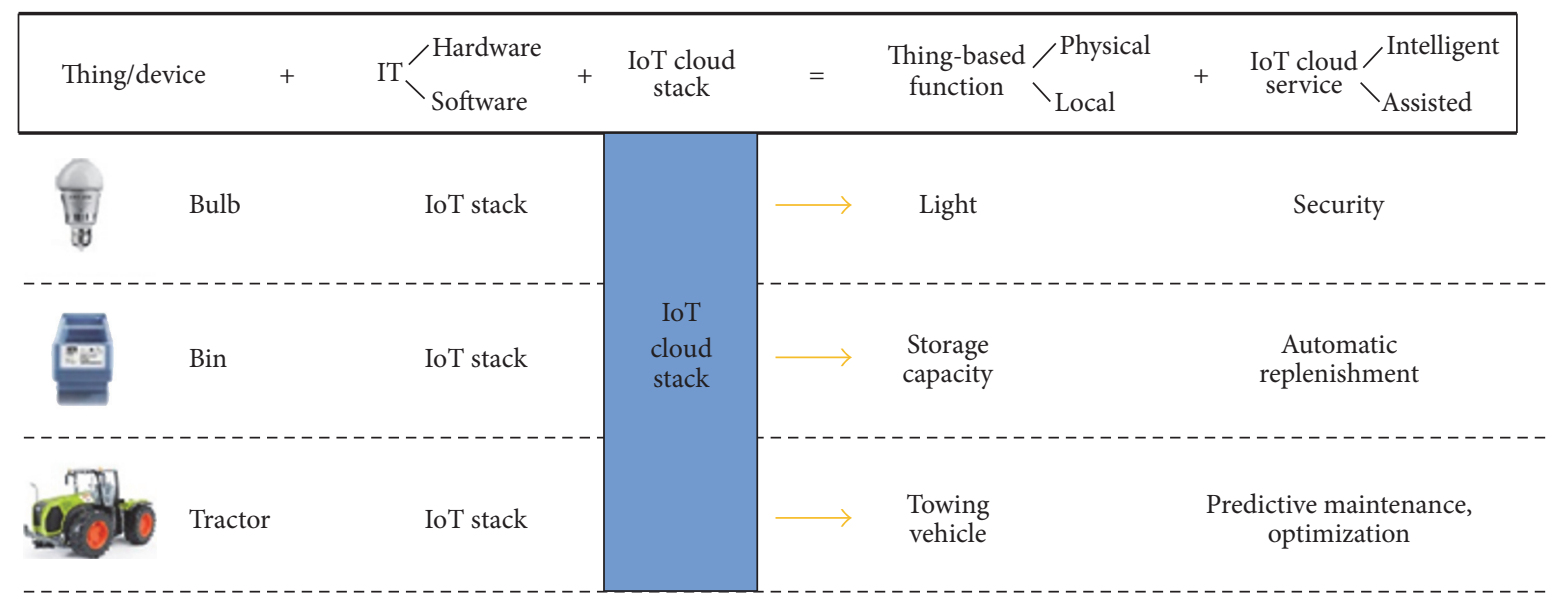

FIGURE 2: IoT cloud based value generation (based on [4]).

farming. However this physical thing-based function when attached to IoT cloud may allow the farmer to know about its predictive nature of maintenance and optimized driving sessions [30].

Not only is the value of things/devices limited to the connected behavior of IoT as presented, but also it may be uplifted to a higher abstraction level of superseded flow of business where a cumulative IoT ecosystem would enhance the basic functionality into a larger context. For instance, a set of IoT cloud served bulbs may create intelligent lighting system, which could include active and passive Infrared (IR) movement detector and heat sensor to extend the overall equipment efficiency of the lighting system. If it goes beyond the ordinary lighting system, it may be turned into a product system that could be attached with standards and upcoming telecommunication technologies, for example, 2G, 3G, LTE (4G); besides Bluetooth Low Energy (BLE) 4.0, IEEE 802.11 $\mathrm{b} / \mathrm{g} / \mathrm{n}-\mathrm{Wi}-\mathrm{Fi}$, even Zigbee antennas could be plugged-in so that the energy consumption and accidental incidents be easily monitored, analyzed, and apprehended.

Data analytics in IoT cloud may improve the system's throughput in optimized manner. This may incur real value in the lighting system that may replenish the necessity of stochastic behavior by strengthening the base of IoT cloud and provide vibration to other sectors of business.

\section{Technologies behind IoT Cloud Stack}

This section describes IoT cloud stack architecture in detail to inculcate the business value generation process from IoT cloud platforms.

It is evident that the proper functioning of businessvalue-incorporated connected device is comprised of a stringent layered stack of relevant technologies as shown in Figure 3. The figure illustrates the principal activities of IoT cloud in a stack form on top of the IoT (which includes things, network connectivity, and middleware and is also supported by security issues while extending the interface of business computing) which lays the foundation for the prescribed system.
The IoT cloud stack is subdivided into four portions such as infrastructure, business applications, business services, and business processes.

In infrastructure module, networking components such as switches, servers, storage devices, routers, virtualization techniques, operating system, and other elements are included, while middleware and unstructured contents are placed over the distributed database.

Next higher level of abstraction is business applications layer that provides the APP based services. Modular (Java based composite/process app), packaged (CRM, ERP, HCM, etc.), and dedicated business support apps (BI, BPM, BAM, etc.) do run over here.

In IaaS layer, software attributed computing, definition, and networking attributes control the resources (hardware/software) for optimization, orchestration, and abstraction.

Cloud operating environment is created on the next higher layer, that is, PaaS, where various APIs are collaborated with the specific business related tasks; the result is obtained from SaaS layer, where collaborative marketplace is numerated by e-commerce and external business ecosystems.

In business services layer, web portal, product catalogue, inventory management, billing, product shipping, and so forth, core components are present.

In business process layer, mainly transaction related operations are performed. Order management, cash handling, transaction, and procurement monitoring tasks get processed. This topmost layer of IoT cloud stack holds interface with business users and customer providing sheer experience without exploring the underlying architecture of IoT based business protocols.

The aforementioned IoT cloud platform is capable of producing value out of connected objects in a modular fashion. Relevant corporate ancillaries act on the discussed IoT platform.

To the author's best knowledge, there is no standard IoT cloud stack available till date; hence a multitude of versions are being opted by the users and business farms. 


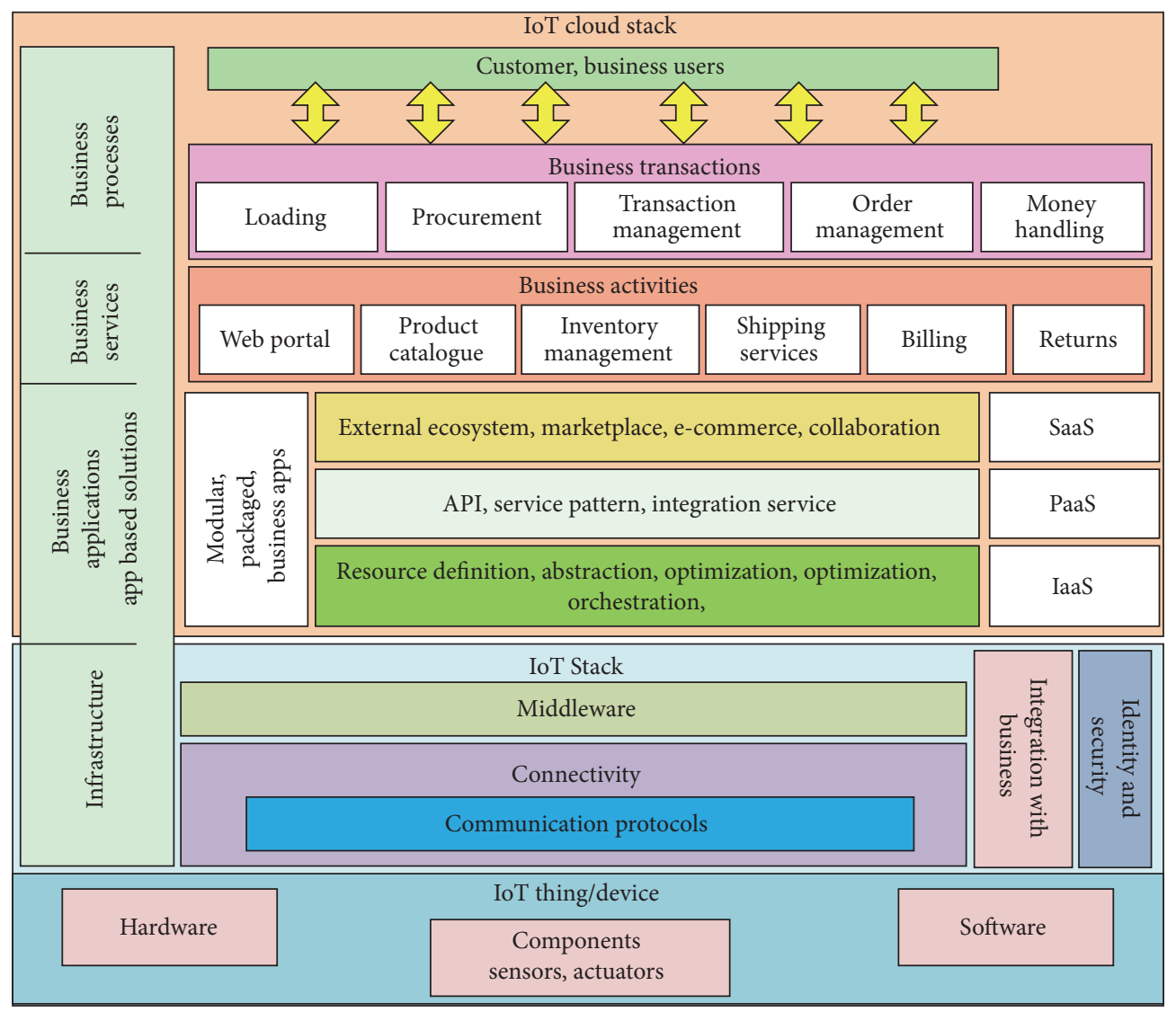

FIGURE 3: IoT cloud stack based on IoT stack.

Each platform varies from the other in terms of protocol inclusion and value generation methodologies.

\section{Challenges Related to Industrial Internet of Things}

Though Industrial Internet of Things (Industrial IoT) has great promising opportunities, many risk factors could hinder its future growth. Not surprisingly, security and interoperability are the two biggest hurdles. Lack of clearly defined Return on Investment (RoI), technology immaturity, legacy equipment, and so forth are among the other significant barriers. Several other issues and challenges are shown in Figure 4. As more physical things come online, vulnerabilities for cyberattacks do also prioritize. Cyberattack may be seen as very or extremely high risk factor associated with Industrial IoT. Privacy breach of personal data is another high risk factor. For example, when both types of risks are justified then one can consider such negative impacts as attacking a power plant to deny electricity to its customers which shall cause loss of public trust and interest resulting in any Industrial Internet-enabled system to be compromised. A recent report published by WTF has estimated around 3 trillion dollars in potential economic loss which may occur from cybersecurity issues by 2020 . This evidently incurs the business investments to take countermeasures against it. According to Gartner, more than $20 \%$ of the existing enterprises will invest in security measures in business initiatives incorporating IoT devices by 2017 [23]. Resulting potential disruption occurring into the existing business models may constitute several related risks for incumbent business players. The gross shifting from products to service oriented outcomes will not only disrupt internal corporate operations but eventually impact the mechanism of how they go to the global market.

Due to the openness in the access and control points among the current digital marketplace, this shall compel the companies to face tough competition from a larger set of key players which may include pure digital players based on innovative business models and the traditional digital platforms who are present from the very inception of computer. This shall levy an opportunity for the companies to have the flexibility to partner with other organizations across the industrial ecosystem. Obviously, such collaboration will harness an imperative impact on the companies which want to meet the ever growing customer expectations around the service or product delivery results. For example, it will be difficult to measure how one enterprise can master the entire digital value chain for which it has developed the product dissemination system. From the societal point of view, it shall be important to consider the potential job displacement ratio that will surely occur in some industrial sectors due to IoT based increased automation. This process is very similar to the communications switchboard job holders who were rapidly replaced by the software based technology solutions. 


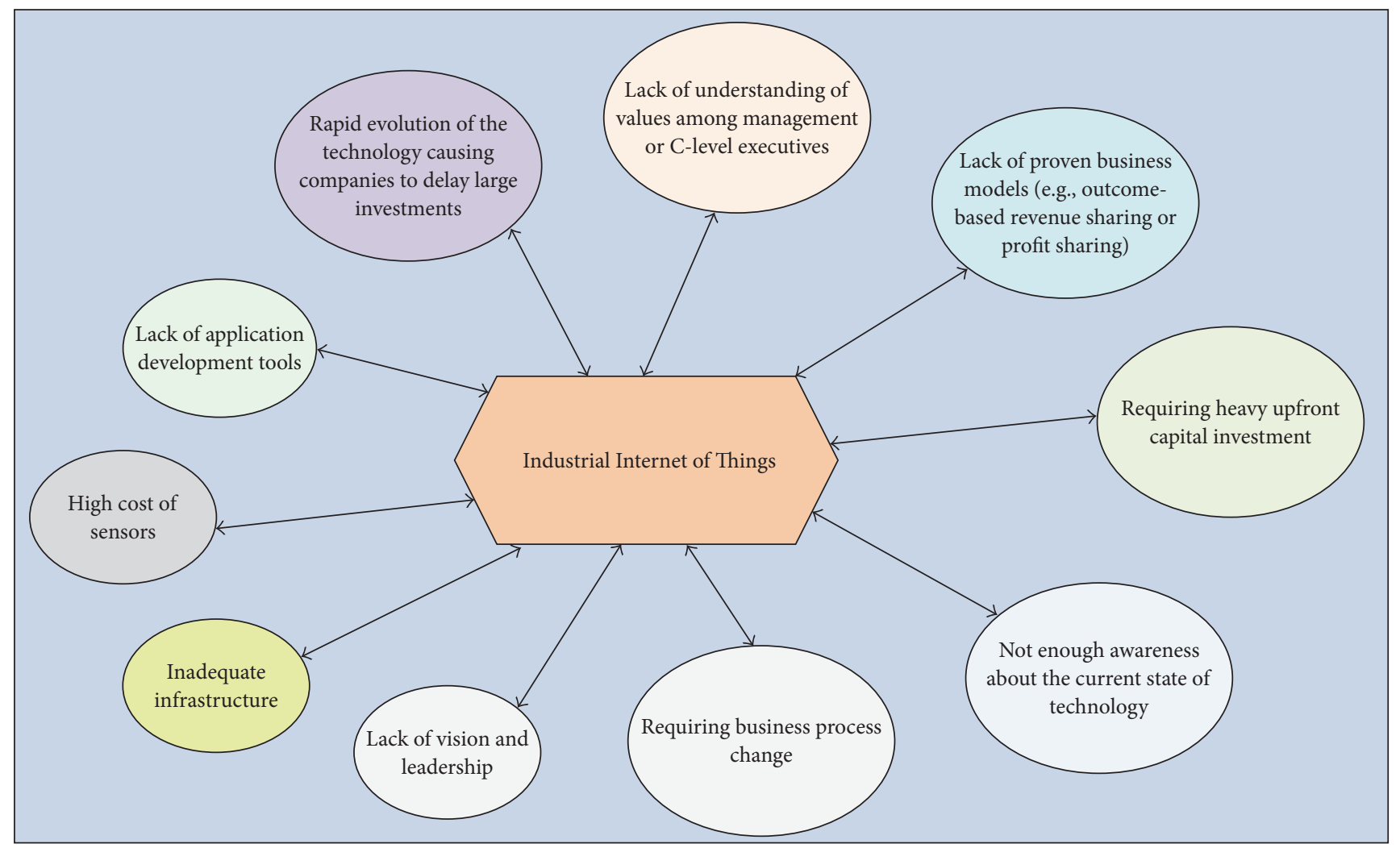

FIGURE 4: Risk factors of Industrial IoT.

With more intelligent machines becoming widespread, more human jobs will be displaced. For instance, if we consider the current pace of technology improvement, maid robots may replace human home maids in the next 20 years. However, we have to anticipate that many new and different types of jobs will be created in due action. Human having uniqueness, such as critical thinking, creativity, and collaboration, shall be employed in future. It is high time that industry and government leaders should understand the fact that improved technology is constantly upgrading the quality bar for the low-skilled jobs. This necessitates strong and urgent need to tackle current issues related to skill-less labor in the IoT based IT market. New action plans are urgently needed to reassess the attention on the current structure of education, adapting with the current propaganda of educational systems, and find innovative approaches to prepare new generations for the upcoming IoT based digital workplace.

Moreover the below-mentioned technological challenges need to be revised and reassessed:

(A) Strategic Level. Here, new and innovative dimensions need to be sought to make better and efficient implementation of IoT cloud stack into the business. Corporate houses should rethink the fundamental questions such as how to cover up the business around the newly developed technologies, how to frame the skeleton of the business rules, and how to adapt the incremental benefits from the stack, so that production be enhanced with less effort.
(B) Operational Level. Initial product development stage may now be redesigned for sake of leveraging space to new segments of improvisations including IoT. Questions such as how to degenerate the initiation of product development in view of smart IoT connected devices, how to integrate the followups with the existing business model, how to negotiate the sales procedure, and how to market the deliverables in advanced variations should be addressed.

(C) Capacity Building. When talking about social involvedness, IoT cloud stack shall revolutionize the human life in more ways than one. New knowledge and skills sets in nearly every field (statistics, engineering, and medicine) should be set in such a way that skilled man power be generated; citizen participation is another factor that should be grown among the people of society towards the hands on experience of IoT; moreover, quality of livelihood needs to be rebuilt while implying efficient urbancivic operations by a guaranteed framework of high yielding economic, societal, and environmental values for future generations.

(D) Technological Level. This area should be better highlighted to make IoT cloud based business process profit yielding by incorporating novel theories, structures, and applications, especially in the subject like computer science and mathematics. 


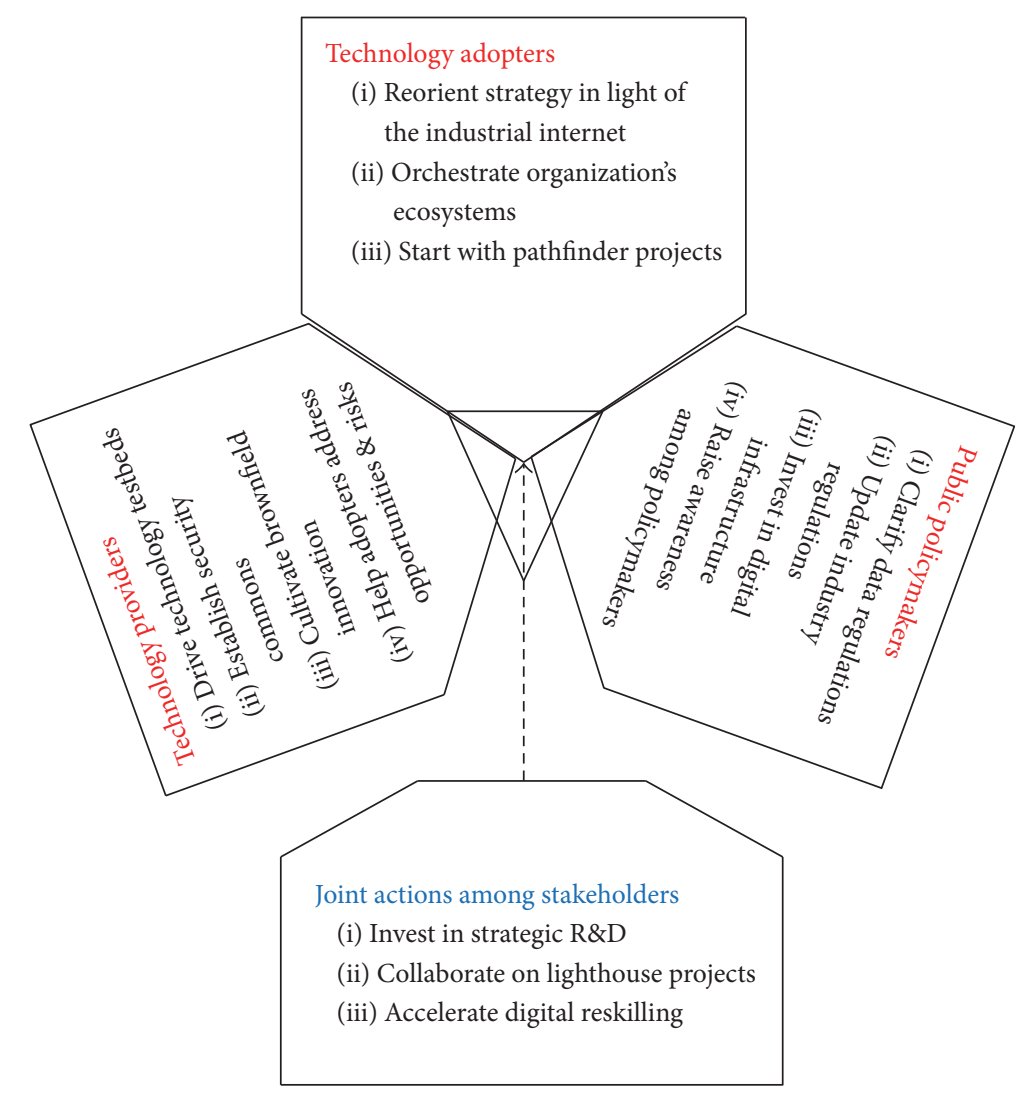

FIGURE 5: Key recommendations for the three stake holders of Industrial IoT sector.

(i) Big Data. High volume, high velocity, high variety, high veracity, and high value $(5 \mathrm{~V})$ conglomerate into a new term called big data problem. Exponential increase of usage patterns of mobile and sensor enabled devices have developed a space where enormous amount of heterogeneous data get accumulated or trafficked through the network every instance of the clock. New methodologies, algorithms, and databases are to be framed to formulate the access and manipulation problems in current scenario.

(ii) ICP. Format identification, data capture, and data processing fields need to be revisited so the IoT enabled connected environments may be facilitated by extracting valuable information from it.

(iii) Security. Privacy, security, and trust issues are no longer a local notion but transborder measure of distributed data exchange and processing. Each device and each people's notion should be maintained to safeguard the proclamations of IoT cloud stack.

\section{Recommendations for Industrial Internet of Things}

As of now, we have discussed the Industrial IoT being already used for delivering actual benefits including improved business operational efficiency, flexibility in work experience, and monitorable and measurable business outcomes. At the same time, we have also pointed out a number of risk factors that might possibly slow down the pace of Industrial IoT development while increasing the various risks of adoption including security, data use policies, interoperability, upgraded education system, and skill gaps. This section provides necessary recommendations made that the business farms and government stakeholders need to take prompt actions so as to regain underlying opportunities, overcome key challenges, and accelerate the growth of Industrial IoT in digital domain. Figure 5 describes the Industrial IoT related interactions between three stakeholders in form of mandates to be asked by them to obtain the correct answer.

\subsection{For Technology Adopters}

(i) Orientation of the Business Strategy. Few questions need to be asked by the technology adopters to themselves for further progression in Industrial IoT such as the following: 
Where is the present situation of the industry among the four-phase Industrial IoT evolution model? (See Figure 5.)

When is the probable next inflection point on business graph?

Business adopters should try to develop multitude of scenarios regarding various possible alternative features and map out the earlier mentioned strategy based on the company's current profile and requirement.

They should identify the exact structure of process as well as organization so as to achieve long-term success by asking how asset utilization can set up the company for future success.

How can we transit between each of the four phases?

What are the probable implications that got originated from existing assets and buyer-seller behavior?

(ii) Define the Organization's Ecosystems. One option is that multiple providers are required to collaborate for delivery of complex outcome while sharing costly investments. Few more questions need to be asked such as the following:

Having multiple product lines, which ecosystem(s) should the farm lead?

Where should the company play a supporting role?

What type of business partners will the company need to merge so as to boost overall capabilities to deliver the expected outcomes?

Will the present partners be the right ones for the future?

What activities shall the business perform to be a collaborative partner?

Who should the company partner with to reach higher phases?

How do we reduce risk?

How do we look across industry boundaries to seek emerging opportunities, so that it can find the potential partners that may help in regaining the scenario?

(iii) Driving Issues of the Business. Company should momentarily imply various issues such as cycle-time reductions, business process improvements, and cost savings to drive near-term measurable benefits. Few questions may be asked to seek the answers such as the following:

How can we balance the implementations of low-cost pilot projects?

Which business model implication is suitable for adaptation?
What kind of outcome-based services can it opt for?

What are the crucial requirements for conducting such pilot project?

How can support from governments at early stage be obtained?

\subsection{For Technology Providers}

(i) Interoperability. Technology providers should seek some questions to ascertain the solutions of the aforementioned problems such as the following:

How can real-world testbeds be developed to concatenate and support new use cases and product?

How can voice be directed in shaping futuristic Industrial IoT products and services?

What are the current trends?

What is the possible timing around probable market disruptions?

How can we bootstrap connected industry ecosystems and platforms?

(ii) Share through a Global Commons. Few questions need to get answered for sharing practice in a secure common means such as the following:

How do the safety and security practices vary across the ecosystem?

How do we step forward towards a common security framework?

How do we identify parameters for cybersecurity products?

How do we design a global security commons?

How can the commons help collective security awareness?

How can a unified industry voice be ensured?

(iii) Cultivate the Innovation. Technology providers need to know few facts by asking questions such as the following:

How and what new devices can be added to improve integrity of the existing machinery?

What components (hardware or software) of the system need to be revised?

What type of value added services can be provided?

What kind of new outcome-based opportunities may be opened?

(iv) Market Opportunities and Risks. The Industrial IoT is still in nascent stage. So, companies should leverage the knowledge by asking the following questions to develop a clear view before they start running on it: 
What amount of benefits has been devised so far?

What are the potential barriers that need to be overcome at the earliest?

What is the knowledge earned from past issues? How can we leverage the most effective ways to adopt experiences?

6.3. For Public Policymakers. Government and public figures shall pave new ways to cultivate Industrial IoT processing in timely manner. Few concepts are given as follows.

(i) Define Data Policies. Policies are a stake to realize the promising potential of Industrial IoT while drafting vivid strategies over data. Related questions to be sought are as follows:

Who will own the data generated by the Industrial Internet-enabled devices?

What type of sized information can be used?

How do we handle the data originated and used in different jurisdiction?

How do we segregate Industrial Internet data from that of consumer Internet?

How should government collaborate with each other around the globe?

What steps need to be taken to harmonize compliance requirements in data among the companies?

(ii) Industry Regulations. Industrial regulations are very much crucial for any government to control monitor over it. Few solutions should be sought out such as the following:

How should industries make benefit out of the Industrial IoT?

How can more flexibility among the company regulations be provided to help them invent?

How would the government use the utility to tap with industry for the sake of new power of transparency?

How can we leverage better customer services among the industries?

Which sector of industries should be regulated to avail security, efficiency, and services to public?

How would insurance regulations be implied over the growth of industries?

(iii) Investment. Infrastructure lays the foundation of success especially in the ubiquitous, broadband, and connectivity based sectors. Developed countries may invest in emerging digitized markets where it will have a unique opportunity by incorporating the Industrial Internet infrastructure. Besides investing in basic infrastructures such as roads, factories, and high rise buildings, state-of-the-art embedded sensors may be implanted on the real segments of life. Smart city is an area which may enable more efficient use of natural resources be it human or natural and provide better public safety and services. Industries along with the government shall seek prioritizing the infrastructure investments to provide long-term strategic benefits to incumbent the wealthy economic growth.

(iv) Awareness. As of now, a large portion of policymakers are not informed about how Industrial IoT might impact on citizens' livelihood, industries workplace, and governments. Similarly, they still do not know what government can perish to enhance the market and economic growth. Hence, it seems like an urgent need to bring the policymakers up to speed on the recent growth of technologies in Industrial IoT and its societal implications and impact on the government services.

\subsection{Joint Actions among Stakeholders}

(i) Invest in Research and Development. The futuristic approach and invasion of Industrial Internet of Things will engage numerous stakeholder efforts in various research fields (e.g., security, reliability, and interoperability). It shall also deliver huge societal benefits (e.g., smart city) to the citizen. However, security is such an issue that industry and government can only solve by getting together. At the same time, addressing these challenges shall need the involvement from academia, government, and industry. Few questions to be answered are as follows:

How can the stakeholders support technology transfer from within?

How can we fund this research among the academics?

(ii) Reskilling Working Generation. The emergence and growth of Industrial IoT will seek the talent gap among the industry workers. Analytical talent pool (including data scientists) is the demand of present time, though most of the current education approaches are equipped with the challenge. Industries and academia must come together to ask basic questions such as the following:

How do we define and implement new educational and training approaches?

How do we reskill the young generation in the classroom and online?

\section{Conclusion}

Value is an important parameter of any business/marketing system. It tells about the functional capabilities of the system. Until recently, value generation from a specific task was concentrated into a certain portion of prescribed rate. The advent 
of IoT has made this path clear while integrating cloud platforms to itself. Things are now easily integrated to perform targeted job easily. In this paper, IoT cloud is proposed to extract more values (multiple functionalities) from a particular thing. This indeed paves the revenue generation process in business activities more beneficial for the organization. A case study is performed to demonstrate the value generation mechanism from the things associated with IoT cloud. Research challenges are discussed to motivate the scientific community to come forward in this regard. Challenges towards successful implementation of Industrial Internet are also investigated. Recommendations are made for technology adaptors, providers, and public policymakers. Suggestions are provided for the joint actions among stakeholders to aggregate the collaborative notions among themselves. This paper truly paves the insightful investigation to create value out of the Industrial Internet of Things.

\section{Competing Interests}

The author declares that he has no competing interests.

\section{References}

[1] IoT Analytics, http://iot-analytics.com/20-internet-of-thingscompanies/.

[2] IDC, The internet of things moves beyond the buzz: worldwide market forecast to exceed $\$ 7$ trillion by 2020, IDC says [press release], http://www.marketwatch.com/story/the-internet-ofmoves-beyond-the-buzz-worldwide-market-forecast-to-exceed7-trillion-by-2020-idc-says-2014-06-03.

[3] ITU-IoT definition, http://www.itu.int/ITU-T/newslog/New+ ITU+Standards+Define+The+Internet + Of + Things + And +

[4] F. Wortmann and K. Fluchter, "Internet of things: technology and value added," Business \& Information Systems Engineering, vol. 57, no. 3, pp. 221-224, 2015.

[5] H. Lasi, P. Fettke, T. Feld, and M. Hoffmann, "Industry 4.0," Business \& Information Systems Engineering, vol. 6, no. 4, pp. 239-242, 2014.

[6] P. P. Ray, "Home Health Hub Internet of Things $\left(\mathrm{H}^{3} \mathrm{IoT}\right)$ : an architectural framework for monitoring health of elderly people," in Proceedings of the IEEE International Conference on Science Engineering and Management Research (ICSEMR '14), pp. 1-3, Chennai, India, November 2014.

[7] P. P. Ray, "Internet of Things for Sports (IoTSport): an architectural framework for sports and recreational activity," in Proceedings of the IEEE International Conference on Electrical, Electronics, Signals, Communication and Optimization (EESCO '15), pp. 1-4, Visakhapatnam, India, January 2015.

[8] S. Sebastian and P. P. Ray, "Development of IoT invasive architecture for complying with health of home," in Proceedings of the International Conference on Computing and Communication Systems (I3CS '15), pp. 79-83, Shillong, India, April 2015.

[9] P. P. Ray, "Internet of things based physical activity monitoring (PAMIoT): an architectural framework to monitor human physical activity," in Proceedings of the IEEE National Conference on Electrical, Electronics, and Computer Engineering (CALCON '14), pp. 32-34, IEEE, Kolkata, India, 2014.

[10] P. P. Ray, "Towards an internet of things based architectural framework for defence," in Proceedings of the IEEE International Conference on Control, Instrumentation, Communication and
Computational Technologie (ICCICCT '15), pp. 411-416, December 2015.

[11] P. P. Ray, "Generic Internet of Things architecture for smart sports," in Proceedings of the IEEE International Conference on Control, Instrumentation, Communication and Computational Technologies (ICCICCT '15), pp. 405-410, December 2015.

[12] P. P. Ray, "Internet of things based physical activity monitoring (PAMIoT): an architectural framework to monitor human physical activity," in Proceedings of the CALCON, pp. 32-34, Kolkata, India, November 2014.

[13] P. Majumder and P. P. Ray, "Hatch-Sens: a theoretical bioinspired model to monitor the hatching of plankton culture in the vicinity of wireless sensor network," International Journal of Computer Science and Information Technologies, vol. 3, no. 4, pp. 4764-4769, 2012.

[14] P. P. Ray, A. Sharma, and R. Rai, "MDTRM: abstraction to model driven tree reference model of internet of things," in Proceedings of the National Conference on Applied Electronics (NCAE '13), pp. 61-64, Adamas Institute of Technology (AIT), West Bengal, India, 2013.

[15] R. Rai, C. Lepcha, P. P. Ray, and P. Chettri, "GDMA: generalized domain model architecture of internet of things," in Proceedings of the National Conference on Applied Electronics (NCAE '13), pp. 65-68, AIT Kolkata, October 2013.

[16] S. M. Babu, A. J. Lakshmi, and B. T. Rao, "A study on cloud based internet of things: CloudIoT," in Proceedings of the Global Conference on Communication Technologies (GCCT '15), pp. 6065, Thuckalay, India, April 2015.

[17] V. C. Emeakaroha, N. Cafferkey, P. Healy, and J. P. Morrison, "A cloud-based iot data gathering and processing platform," in Proceedings of the 3rd International Conference on Future Internet of Things and Cloud (FiCloud '15), pp. 50-57, IEEE, Rome, Italy, August 2015.

[18] B. Kantarci and H. T. Mouftah, "Sensing services in cloudcentric internet of things: a survey, taxonomy and challenges," in Proceedings of the IEEE International Conference on Communication Workshop (ICCW' 15), pp. 1865-1870, London, UK, June 2015.

[19] A. Botta, W. De Donato, V. Persico, and A. Pescapé, "On the integration of cloud computing and internet of things," in Proceedings of the 2nd International Conference on Future Internet of Things and Cloud (FiCloud '14), pp. 23-30, IEEE, Barcelona, Spain, August 2014.

[20] G. Suciu, A. Vulpe, O. Fratu, and V. Suciu, "M2M remote telemetry and cloud IoT big data processing in viticulture," in Proceedings of the 11th Intercenational Wireless Communications and Mobile Computing Conferen (IWCMC '15), pp. 1117-1121, IEEE, Dubrovnik, Croatia, August 2015.

[21] L. Wang and R. Ranjan, "Processing distributed internet of things data in clouds," IEEE Cloud Computing, vol. 2, no. 1, pp. 76-80, 2015.

[22] S. Nastic, S. Sehic, D.-H. Le, H.-L. Truong, and S. Dustdar, "Provisioning software-defined IoT cloud systems," in Proceedings of the 2nd International Conference on Future Internet of Things and Cloud (FiCloud '14), pp. 288-295, August 2014.

[23] S. Nastic, G. Copil, H. Truong, and S. Dustdar, "Governing elastic IoT cloud systems under uncertainty," in Proceedings of the IEEE 7th International Conference on Cloud Computing Technology and Science, pp. 131-138, Vancouver, Canada, 2015.

[24] H. Truong and S. Dustdar, "Principles for engineering IoT cloud systems," IEEE Cloud Computing, vol. 2, no. 2, pp. 68-76, 2015. 
[25] S. W. Kum, J. Moon, T. Lim, and J. I. Park, "A novel design of IoT cloud delegate framework to harmonize cloud-scale IoT services," in Proceedings of the IEEE International Conference on Consumer Electronics (ICCE '15), pp. 247-248, Las Vegas, Nev, USA, January 2015.

[26] A. Celesti, M. Fazio, M. Giacobbe, A. Puliafito, and M. Villari, "Characterizing cloud federation in IoT," in Proceedings of the 30th International Conference on Advanced Information Networking and Applications Workshops, pp. 93-98, 2016.

[27] A. Taherkordi and F. Eliassen, "Scalable modeling of cloudbased IoT services for smart cities," in Proceedings of the 1st IEEE International Workshop on Context-Aware Smart Cities and Intelligent Transport Systems, Sydney, Australia, March 2016.

[28] G. Fortino, A. Guerrieri, W. Russo, and C. Savaglio, "Integration of agent-based and cloud computing for the smart objectsoriented IoT," in Proceedings of the 18th IEEE International Conference on Computer Supported Cooperative Work in Design (CSCWD '14), pp. 493-498, IEEE, Hsinchu, Taiwan, May 2014.

[29] P. P. Ray, "Internet of things cloud enabled MISSENARD index measurement for indoor occupants," Measurement, vol. 92, pp. 157-165, 2016.

[30] E. Fleisch, What is the internet of things-an economic perspective, Auto-ID labs white paper, 2014, http://cocoa.ethz.ch/ downloads/2014/06/None_AUTOIDLABS-WP-BIZAPP-53.pdf. 


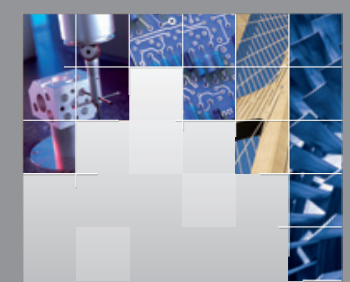

\section{Enfincering}
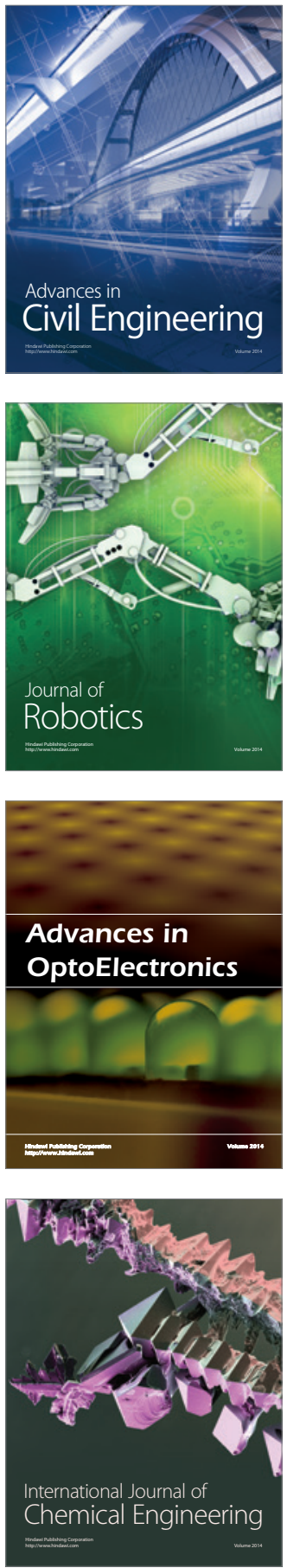

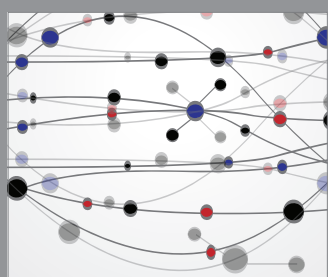

The Scientific World Journal

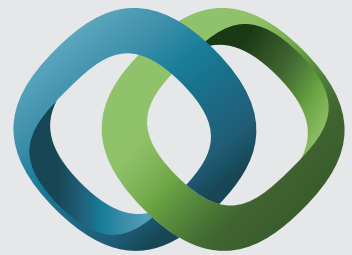

\section{Hindawi}

Submit your manuscripts at

http://www.hindawi.com
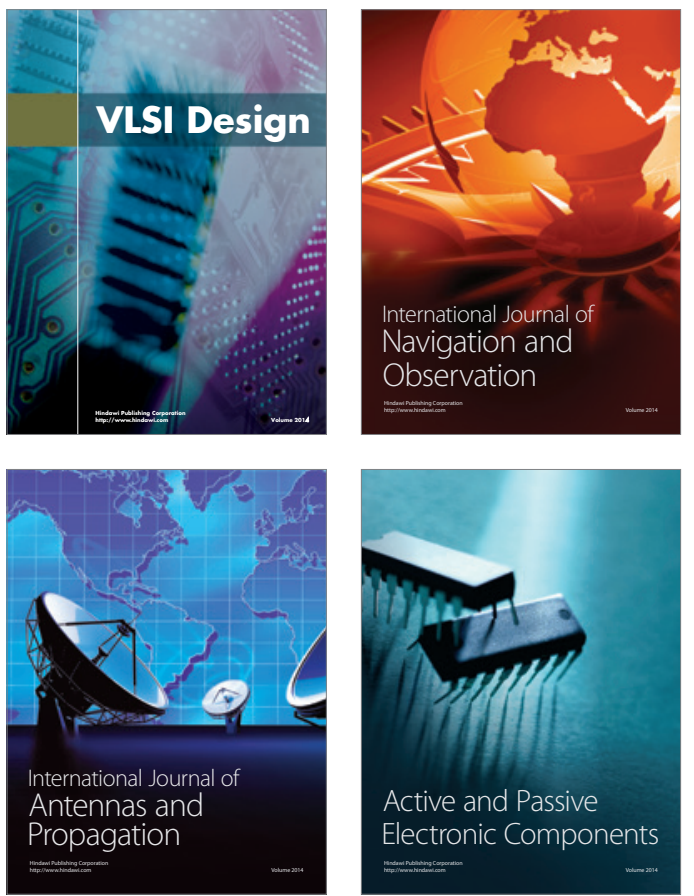
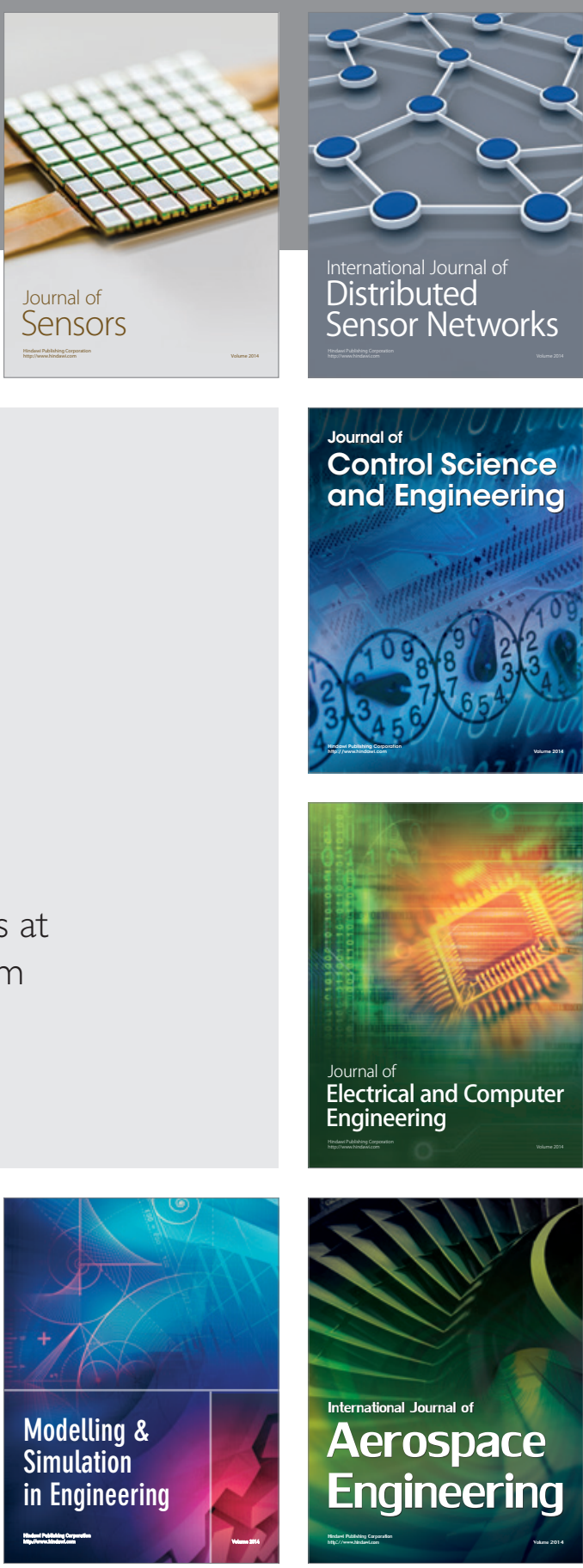

International Journal of

Distributed

Sensor Networks

Journal of

Control Science

and Engineering
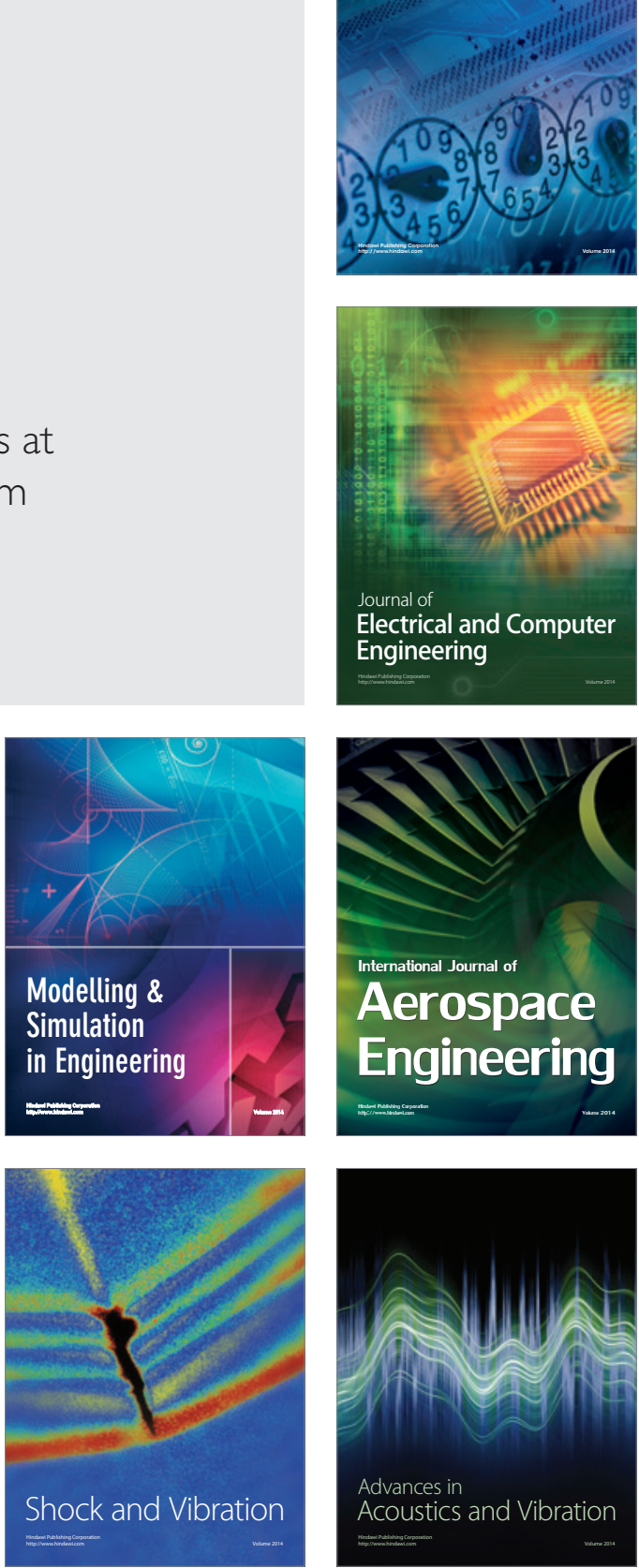